“( 2014 IEEE. Personal use of this material is permitted. Permission from IEEE must be obtained for all other uses, in any current or future media, including

reprinting/republishing this material for advertising or promotional purposes, creating new collective works, for resale or redistribution to servers or lists, or reuse of any copyrighted component of this work in other works." 


\title{
Controllability Analysis of the First FM Model of 2D Systems: A Row (Column) Process
}

\author{
Ahmadreza Argha, Li Li, Steven W. Su* and Hung Nguyen
}

\begin{abstract}
Dealing with 1D form of 2D systems is an alternative strategy to reduce the intrinsic complexity of $2 \mathrm{D}$ systems and their applications. To obtain the 1D form of 2D systems, a row (column) process is used in this paper. The controllability analysis of the obtained 1D form and its relation to the local controllability of the local states in the original 2D system is the subject of this paper. Moreover, in this paper, a new notion of controllability named directional controllability is defined and studied for the underlying $2 \mathrm{D}$ systems.
\end{abstract}

\section{INTRODUCTION}

Different time domain analysis, such as controllability, reachability and observability of $2 \mathrm{D}$ systems, has been considered so far, and some notions such as local, global and causal controllability (reachability) are defined for 2D systems; e.g. see [1], [2], [3], [4], [14] - [16], [19], [17]. Necessary and sufficient conditions for the exact reconstructibility of the state of the second FM model have been presented in [17]. Another necessary and sufficient conditions with respect to $2 \mathrm{D}$ matrix polynomial equations for the local controllability and the causal reconstructibility of 2D linear systems are proposed in [18]. Reference [15] has defined notions of the local controllability, reachability, and reconstructibility for the general singular model of $2 \mathrm{D}$ linear systems.

Moreover, the theory of 2D systems has been used for the special problem of repetitive linear processes [10] - [12]. Indeed, in repetitive processes, the systems make a series of sweeps through a set of dynamics defined over a finite duration. Once each iteration (pass) is complete, the system resets to the starting location and on the next sweep the output on the previous step, explicitly, contributes to the output on the current one [11]. Some new notions of point controllability and pass controllability are defined in [10], [11].

A different technique to deal with 2D systems is to transfer the underlying 2D system to $1 \mathrm{D}$ form. Wave advance model (WAM) is a 1D form of 2D systems established by [5]. In WAM model, the 2D systems are considered as advanced waves and consequently the original stationary 2D system is converted to a time varying 1D system. Moreover, the system matrices are in rectangular form rather than square form. As a result, the major drawback of this 1D form of $2 \mathrm{D}$ systems is the varying dimensions of the defined state vectors. This matter makes it hard and probably impossible

\footnotetext{
Ahmadreza Argha, Li Li, Steven W. Su and Hung Nguyen are with Faculty of Engineering and Information Technology, University of Technology, Sydney, PO Box 123, Broadway, NSW 2007, Australia.

\{Ahmadreza.Argha, Li.Li, Steven.Su, Hung. Nguyen\} euts. edu.au
}

to adopt different kind of control methods [10] and, in particular, discrete-time sliding mode control (DSMC) to the obtained 1D form [9]. However, using stacking vectors, a new method is proposed in [10] - [12] to convert 2D repetitive linear discrete-time processes to a $1 \mathrm{D}$ form and, in addition, for the first FM model in [9]. Hence, rather than using WAM method, a row (column) processing method is used. Row (column) processing means that the 2D local states which are in the same rows (columns) are used to form 1D stacking vectors. Consequently, the states, inputs and outputs of the obtained 1D system are in the vector form, and more importantly their dimensions are invariant. This method is basically useful for a class of 2D linear systems in which information propagation in one of the two distinct directions only occurs over a finite horizon. This can be a repetitive process [10] or any inherently 2D system, for instance, Darboux equation [13]. By means of illustration, the discrete form of Darboux equation which describes the dynamical processes such as gas absorption, water stream heating and air drying, is a first FM model which has finite propagation on the space direction.

On the other hand, during the procedure of designing the sliding surface in [9], it is necessary that the fictitious reduced order system matrix pair or equivalently the original system matrix pair is controllable; e.g. see [6]. But, the controllability of the obtained $1 \mathrm{D}$ form and its relation to the original 2D system is an unanswered problem in [9]. Hence, motivated by this issue, in this paper, the controllability analysis of the proposed method of [9] is given. To this end, a new notion of controllability named directional controllability is introduced and studied for the underlying 2D systems. It should be noted that the result of this paper may be of great importance for those who want to control the 2D systems via 1D frameworks.

The rest of this paper is as follows: in the next section the proposed procedure of converting a first FM model to $1 \mathrm{D}$ vectorial form is introduced. The controllability analysis is represented in Section III. In Section IV, the numerical example is given. Finally, Section V concludes this paper.

\section{1D FORM OF THE FIRST FM MODEL VIA STACK VECTORS}

In this section, firstly, our proposed 1D form of first FM model in [9] is reviewed. Besides, to avoid the direct computation of the inverse of the descriptor matrix, inspired by some related studies on the explicit inverse of block bi-diagonal Toeplitz state matrices, a numerical solution is given. To illustrate how 2D systems can be converted to $1 \mathrm{D}$ 
form, we consider the first FM model with the following formulation,

$$
\begin{aligned}
x(i+1, j+1)=A_{1} x(i+1, j) & +A_{2} x(i, j+1) \\
& +A_{0} x(i, j)+B u(i, j),
\end{aligned}
$$

where $x \in R^{n}$ and $u \in R^{m}$ are respectively local state and control input. In addition, the matrices in this equation are $A_{1} \in R^{n \times n}, A_{2} \in R^{n \times n}, A_{0} \in R^{n \times n}$ and $B \in R^{n \times m}$. It can be seen that this relation is a second order recursive equation. In the next subsection, a brief review of WAM model of 2D systems is given based on the first FM model. Secondly, the drawbacks of this method are explained. These drawbacks motivate us to investigate an alternative 1D form of $2 \mathrm{D}$ systems which is more effective and efficient. Finally, our proposed 1D form of first FM model is presented.

\section{A. WAM model of $2 D$ systems}

Authors of [5] are the first investigators who have considered 2D systems as a 1D model. In this model, by using a different classification on local state vectors of a 2D system, a novel form of local state vectors is achieved. However, as mentioned, this proposed form has varying dimension of states, and coefficient matrices should be updated at each step. To resolve the varying dimension of state vectors in WAM model, in [5] it is suggested to expand all states to the largest dimension via augmenting system matrices with appropriate blocks of zeros. However, the system matrices are still left varying and complicated to compute. Moreover, the framework of obtaining the stacking state vectors, especially for second order 2D models such as the first FM model, is relatively confusing and time-consuming. To illustrate, define the state vectors $\phi(k)$ and $v(k)$ as

$$
\phi(k)=\left[\begin{array}{c}
x(k, 0) \\
x(k-1,1) \\
\vdots \\
x(0, k)
\end{array}\right], v(k)=\left[\begin{array}{c}
u(k, 0) \\
u(k-1,1) \\
\vdots \\
u(0, k)
\end{array}\right] .
$$

In this case, the resulting WAM form of first FM model is as follows

$$
\begin{aligned}
\phi(k+1)=M(k) \phi(k) & +N(k-1) \phi(k-1) \\
& +E(k-1) v(k-1) .
\end{aligned}
$$

Here, matrices $M(k), N(k-1)$ and $E(k-1)$ are determined in the following forms

$$
\begin{aligned}
M(k) & =\left[\begin{array}{c}
I_{k+1} \\
0_{1 \times(k+1)}
\end{array}\right] \otimes A_{2}+\left[\begin{array}{c}
0_{1 \times(k+1)} \\
I_{k+1}
\end{array}\right] \otimes A_{1}, \\
N(k-1) & =T(k) \otimes A_{0}, \quad E(k-1)=T(k) \otimes B,
\end{aligned}
$$

where

$$
T(k)=\left[\begin{array}{c}
0_{1 \times k} \\
I_{k} \\
0_{1 \times k}
\end{array}\right],
$$

and $I_{k}$ is the identity matrix of order $k$. According to (3) it is obvious that by defining the following relation

$$
r(k)=N(k-1) \phi(k-1)+E(k-1) v(k-1),
$$

the relation (3) is converted to a 1D state space model,

$$
\left[\begin{array}{c}
\phi(k+1) \\
r(k+1)
\end{array}\right]=\left[\begin{array}{cc}
M(k) & I \\
N(k) & 0
\end{array}\right]\left[\begin{array}{c}
\phi(k) \\
r(k)
\end{array}\right]+\left[\begin{array}{c}
0 \\
E(k)
\end{array}\right] v(k) .
$$

Remark 1: As can be seen, state vector in (7) is a linear combination of local states and inputs. However, in some applications, having state space equations with direct access to the local states is required. In this case, by introducing a new state vector,

$$
\begin{aligned}
& \hat{\phi}(k)=[x(k, 0), x(k, 1), x(k-1,1), \\
& \quad x(k-1,2), \ldots, x(1, k-1), x(1, k), x(0, k)]^{T},
\end{aligned}
$$

a 1D sate space equation with direct access to the state vectors $\phi(k)$ and $\phi(k+1)$ is acquired.

Remark 2: In the definition of state vectors (8), instead of using local states just on the line $i+j=k+1$, local states located on one the line $i+j=k$ are also used to form state vectors. Generally, for WAM description of 2D systems which are at least second order, using state vector (8) is useful. However, obtaining WAM method for second order 2D systems (for instance FM model) and especially for large scale 2D systems is complicated and, more importantly, the dimension of state vector (8) is varying. On the other hand, as it is explained in [9], to design sliding matrices using regular form based method, it is necessary to find the regular form of state space equation [7], while by using WAM form a new regular form should be found in each step which would result in heavy computational burden.

Remark 3: In the case that the boundary conditions are assumed to be constant, the state vector (8) should get rid of the boundary condition terms $x(k, 0)$ and $x(0, k)$. In other words, the state vector (8) is changed to

$$
\begin{aligned}
& \bar{\phi}(k)=[x(k, 1), x(k-1,1), \\
& \quad x(k-1,2), \ldots, x(1, k-1), x(1, k)]^{T},
\end{aligned}
$$

Hence, the 1D model is as follows

$$
\bar{\phi}(k+1)=\bar{M}(k) \bar{\phi}(k)+\bar{E}(k) v(k)+\bar{V}(k),
$$

where

$$
\begin{gathered}
\bar{M}(k)=\left[\begin{array}{ccccccc}
A_{2} & 0 & 0 & 0 & 0 & \cdots & 0 \\
I & 0 & 0 & 0 & 0 & \cdots & 0 \\
A_{1} & A_{0} & A_{2} & 0 & 0 & \cdots & 0 \\
0 & 0 & I & 0 & 0 & \cdots & 0 \\
0 & 0 & A_{1} & A_{0} & A_{2} & \cdots & 0 \\
\vdots & \vdots & \vdots & & & \cdots & \vdots \\
0 & 0 & 0 & 0 & 0 & \cdots & I \\
0 & 0 & 0 & 0 & 0 & \cdots & A_{1}
\end{array}\right], \\
\bar{E}(k)=\left[\begin{array}{ccccc}
B & 0 & 0 & \cdots & 0 \\
0 & 0 & 0 & \cdots & 0 \\
0 & B & 0 & \cdots & 0 \\
0 & 0 & 0 & \cdots & 0 \\
\vdots & \vdots & \vdots & \cdots & \vdots \\
0 & 0 & 0 & \cdots & B
\end{array}\right]
\end{gathered}
$$

and,

$$
\bar{M}(k) \in R^{[(2 k+1) \cdot n] \times[(2 k-1) \cdot n]}, \quad \bar{E}(k) \in R^{[(2 k+1) \cdot n] \times[(k+1) \cdot m]} .
$$


Moreover, the vector $V(k)$ which arises from boundary conditions is in the following form

$$
\bar{V}(k)=\left[\begin{array}{c}
A_{1} x(k+1,0)+A_{0} x(k, 0) \\
0 \\
\vdots \\
0 \\
A_{2} x(0, k+1)+A_{0} x(0, k)
\end{array}\right],
$$

where $\bar{V}(k) \in R^{(2 k+1) \cdot n}$.

\section{B. New $1 D$ form of $2 D$ first FM model} form

The FM model (1) can be represented in the following

$$
\begin{aligned}
x(i+1, j+1)-A_{1} x(i+1, j) & =A_{2} x(i, j+1) \\
& +A_{0} x(i, j)+B u(i, j) .
\end{aligned}
$$

Now, we define the following stacking vectors

$$
\begin{gathered}
V(i)=\left[\begin{array}{c}
A_{1} x(i+1,0)+A_{0} x(i, 0) \\
0 \\
\vdots \\
0
\end{array}\right], \\
X(i)=\left[\begin{array}{c}
x(i, 1) \\
x(i, 2) \\
\vdots \\
x(i, v)
\end{array}\right], U(i)=\left[\begin{array}{c}
u(i, 0) \\
u(i, 1) \\
\vdots \\
u(i, v-1)
\end{array}\right],
\end{gathered}
$$

where $v$ is the dimension of distinct variable $\{j\}$, moreover, $X(i) \in R^{v \cdot n}, V(i) \in R^{v \cdot n}$ and $U(i) \in R^{v \cdot m}$. As a result, the 2D equation (13) can be presented as

$$
J X(i+1)=K X(i)+L U(i)+V(i)
$$

where

$$
\begin{aligned}
J & =\left[\begin{array}{cccccc}
I & 0 & 0 & \ldots & 0 & 0 \\
-A_{1} & I & 0 & \ldots & 0 & 0 \\
0 & -A_{1} & I & \ldots & 0 & 0 \\
\vdots & \vdots & & \ddots & \vdots & \vdots \\
0 & 0 & 0 & \ldots & I & 0 \\
0 & 0 & 0 & \ldots & -A_{1} & I
\end{array}\right], \\
K & =\left[\begin{array}{cccccc}
A_{2} & 0 & 0 & \ldots & 0 & 0 \\
A_{0} & A_{2} & 0 & \ldots & 0 & 0 \\
0 & A_{0} & A_{2} & \ldots & 0 & 0 \\
\vdots & \vdots & & \ddots & \vdots & \vdots \\
0 & 0 & 0 & \ldots & A_{2} & 0 \\
0 & 0 & 0 & \ldots & A_{0} & A_{2}
\end{array}\right], \\
L & =\left[\begin{array}{cccc}
B & 0 & \ldots & 0 \\
0 & B & \ldots & 0 \\
\vdots & \vdots & \ddots & \vdots \\
0 & 0 & \ldots & B
\end{array}\right] .
\end{aligned}
$$

Here, $x(i+1,0)$ and $x(i, 0)$ are state boundary conditions on boundary $(j=0)$. Moreover, as seen in the vectorial definition (14), the variable $\{j\}$ is hidden in the new defined 1D form. The model in (15) is also known as descriptor model.

In the case that the elements of matrix $J$ are varying and in every step the inverse of this matrix should be computed, a very heavy computational load could happen, especially for 2D grids with large dimensions. However, in our case, the matrix $J$ is constant and consequently, the inverse matrix can be computed only once when applied to DSMC; see [9] for details.

To have the standard form of a 1D Discrete MIMO system, left multiply (15) by $J^{-1}$ to obtain

$$
\Sigma_{v}: X(i+1)=\hat{K} X(i)+\hat{L} U(i)+\hat{R} V(i),
$$

where

$$
\hat{K}=J^{-1} K, \hat{L}=J^{-1} L, \text { and } \hat{R}=J^{-1} .
$$

As seen, in this new 1D form, the dimension of state vectors is constant and consequently finding regular form of state space (15) is possible. This possibility sets the stage for designing specific 1D DSMC for the obtained 1D state space model (17), which is applied in [9].

Remark 4: As mentioned before, the proposed method of this paper needs one of the distinct variables of the underlying 2D systems to be of finite dimension. Moreover, the computing limitations have made it inevitable to assume finite dimensions for both separate directions of 2D systems. Consequently, in this paper, the dimensions of the considered $2 \mathrm{D}$ system is assumed to be $\mu \times v$ and, as a result, the size of $1 \mathrm{D}$ state vector $X(i)$ and control input vector $U(i)$ in (15) are $v \cdot n$ and $v \cdot m$, respectively. Besides, there are two set of boundary conditions $(i=0$ and $j=0$ ).

$$
\begin{cases}\alpha(i)=x(i, 0) & \text { over } j=0, \\ \beta(j)=x(0, j) & \text { over } i=0 .\end{cases}
$$

\section{Controllability Analysis}

In this section, different notions of controllability of 2D system (1) are considered, namely, local controllability and directional controllability. This is achieved by studying the relation between the controllability of the obtained $1 \mathrm{D}$ system (17) and that of 2D system (1).

\section{A. Notion of local controllability for 2D system}

In the literature, one can find a number of different definitions of controllability according to different type of dynamical system. Broadly speaking, considering the controllability of $2 \mathrm{D}$ systems is relatively more complex compared to $1 \mathrm{D}$ systems. Instead of notion of controllability which is introduced for 1D discrete-time systems, notion of local controllability (reachability) is developed for 2D systems [8]. Here, the controllability of the first FM model (1) is studied referring to [3] and [8].

To this end, define the so called state transition matrix $A^{i, j}$ as

$$
\begin{aligned}
A^{i, j} & =A_{0} A^{i-1, j-1}+A_{1} A^{i, j-1}+A_{2} A^{i-1, j} \\
& =A^{i-1, j-1} A_{0}+A^{i, j-1} A_{1}+A^{i-1, j} A_{2}, \quad \forall i, j>0 .
\end{aligned}
$$

Furthermore, it is assumed that

$$
A^{0,0}=I_{n}, A^{-i, j}=A^{i,-j}=A^{-i,-j}=0, \quad \forall i, j>0 .
$$


Hence, with boundary conditions (18) and given admissible controls sequence, it can be shown

$$
\begin{aligned}
x(i, j) & =A^{i-1, j-1} A_{0} x(0,0) \\
& +\sum_{p=1}^{i}\left(A^{i-p, j-1} A_{1}+A^{i-p-1, j-1} A_{0}\right) x(p, 0) \\
& +\sum_{q=1}^{j}\left(A^{i-1, j-q} A_{2}+A^{i-1, j-q-1} A_{0}\right) x(0, q) \\
& +\sum_{p=0}^{i-1} \sum_{q=0}^{j-1} A^{i-p-1, j-q-1} B u(p, q) .
\end{aligned}
$$

In this case, we have

$$
\begin{aligned}
M(i, j) & \triangleq x(i, j)-A^{i-1, j-1} A_{0} x(0,0) \\
& -\sum_{p=1}^{i}\left(A^{i-p, j-1} A_{1}+A^{i-p-1, j-1} A_{0}\right) x(p, 0) \\
& -\sum_{q=1}^{j}\left(A^{i-1, j-q} A_{2}+A^{i-1, j-q-1} A_{0}\right) x(0, q) \\
& =\sum_{p=0}^{i-1} \sum_{q=0}^{j-1} A^{i-p-1, j-q-1} B u(p, q) \\
& =C_{i j} u_{i j}
\end{aligned}
$$

where

$$
\begin{array}{r}
C_{i j}=\left[A^{i-1, j-1} B, A^{i-1, j-2} B, \cdots, A^{i-1,0} B, \cdots,\right. \\
\left.A^{0, j-1} B, A^{0, j-2} B, \cdots, B\right],
\end{array}
$$

and

$$
\begin{gathered}
u_{i j}=\left[u^{T}(0,0), u^{T}(0,1), \cdots, u^{T}(0, j-1), \cdots,\right. \\
\left.u^{T}(i-1,0), u^{T}(i-1,1), \cdots, u^{T}(i-1, j-1)\right]^{T} .
\end{gathered}
$$

Definition 1: Consider system (1) with boundary conditions (18). This system is locally controllable in a given rectangle $[(0,0),(\mu, v)]$ if for every boundary conditions (18) and for every vector $x_{d} \in R^{n}$, there exists a sequence of controls $u_{\mu v}$ as in (24) such that $x(\mu, v)=x_{d}$.

Remark 5: The matrix $C_{i j}$ in (23) is known as local controllability matrix.

Lemma 1 ( [8]): System (1) is locally controllable in a given rectangle $[(0,0),(\mu, v)]$ with unconstrained control inputs $u$ if and only if $\operatorname{rank}\left(C_{\mu v} \cdot C_{\mu v}^{T}\right)=n$.

Furthermore, it is shown in [3] that Lemma 1 can be confined to the following lemma.

Lemma 2 ( [3]): System (1) is locally controllable in a given rectangle $[(0,0),(\mu, v)]$ with unconstrained control inputs $u$ if and only if $\operatorname{rank}\left(C_{n n} \cdot C_{n n}^{T}\right)=n$ where $\mu \geq n$ and $v \geq n$.

Remark 6: It should be mentioned that this lemma is proven in Lemma 4.1 and Proposition 4.1 of [3]. Note that the transition matrix $A^{i, j}$, here, is equal to $M_{j i}$ of [3].

\section{B. Directional controllability with respect to $\{j\}$-direction}

In this subsection, the controllability of the $1 \mathrm{D}$ system in (17) is considered. Moreover, a new notion of controllability for this special form of 2D system in (1) is defined. Now, define

$$
\mathscr{M}(i)=\left[\begin{array}{c}
M(i, 1) \\
M(i, 2) \\
\vdots \\
M(i, v)
\end{array}\right]=\left[\begin{array}{c}
C_{i 1} u_{i 1} \\
C_{i 2} u_{i 2} \\
\vdots \\
C_{i v} u_{i v}
\end{array}\right] .
$$

Since $u_{i 1}, u_{i 2}, \ldots, u_{i(v-1)}$ are included in $u_{i v}$, (25) can be rewritten as

$$
\mathscr{M}(i)=\mathscr{C}_{i} u_{i v},
$$

where $\mathscr{C}_{i}$ is the matrix in (27) shown at the top of the next page.

Lemma 3: The matrix $\mathscr{C}_{i}$ in (27) is equivalent to

$$
\mathscr{C}_{i}=\left[\hat{K}^{i-1} \hat{L}|\cdots| \hat{K} \hat{L} \mid \hat{L}\right],
$$

where $\hat{K}, \hat{L}$ (defined in (17)) are as matrices in (35) at the top of the second next page.

Proof: It can be shown that $A^{0, r}=A_{1}^{r}$ and $A^{r, 0}=A_{2}^{r}$. Hence, matrix $\hat{L}$ can be rewritten as,

$$
\hat{L}=\left[\begin{array}{ccccc}
B & 0 & \cdots & 0 & 0 \\
A^{0,1} B & B & \cdots & 0 & 0 \\
\vdots & \vdots & & \vdots & \vdots \\
A^{0, \nu-1} B & A^{0, \nu-2} B & \cdots & A^{0,1} B & B
\end{array}\right] .
$$

Now, assuming

$$
\begin{aligned}
& \hat{K}^{r-1} \hat{L}= \\
& {\left[\begin{array}{ccccc}
A^{r-1,0} B & 0 & \cdots & 0 & 0 \\
A^{r-1,1} B & A^{r-1,0} B & \cdots & 0 & 0 \\
\vdots & \vdots & & \vdots & \vdots \\
A^{r-1, \nu-1} B & A^{r-1, \nu-2} B & \cdots & A^{r-1,1} B & A^{r-1,0} B
\end{array}\right],}
\end{aligned}
$$

then, it can be found that

$$
\hat{K}^{r} \hat{L}=\left[\begin{array}{ccccc}
A^{r, 0} B & 0 & \cdots & 0 & 0 \\
A^{r, 1} B & A^{r, 0} B & \cdots & 0 & 0 \\
\vdots & \vdots & & \vdots & \vdots \\
A^{r, v-1} B & A^{r, v-2} B & \cdots & A^{r, 1} B & A^{r, 0} B
\end{array}\right] .
$$

Remark 7: Besides, $\mathscr{C}_{i}$ can be obtained directly. To this end, from (17), it can be demonstrated that

$$
X(i)-\hat{K}^{i} X(0)-\hat{\mathscr{C}}_{i} \mathscr{V}(i)=\mathscr{C}_{i} \mathscr{U}(i),
$$

where

$$
\mathscr{V}(i)=\left[\begin{array}{c}
V(0) \\
V(1) \\
\vdots \\
V(i-1)
\end{array}\right], \mathscr{U}(i)=\left[\begin{array}{c}
U(0) \\
U(1) \\
\vdots \\
U(i-1)
\end{array}\right],
$$

and $U, V$ are defined in (14). Moreover, $\hat{\mathscr{C}}_{i}$ is obtained from $\mathscr{C}_{i}$ in (27), by replacing $B$ with $I_{n}$. Moreover, it is crystal clear that $\mathscr{U}(i)=u_{i v}$.

As seen, the matrix $\mathscr{C}_{i}$ has the form of the controllability matrix of 1D system (17), hence, the controllability of 1D system (17) can be analyzed by checking the rank of this 


$$
\mathscr{C}_{i}=\left[\begin{array}{ccccc|c|ccccc}
A^{i-1,0} B & 0 & \cdots & 0 & 0 & \cdots & B & 0 & \cdots & 0 & 0 \\
A^{i-1,1} B & A^{i-1,0} B & \cdots & 0 & 0 & \cdots & A^{0,1} B & B & \cdots & 0 & 0 \\
\vdots & \vdots & & \vdots & \vdots & \vdots & \vdots & \vdots & & \vdots & \vdots \\
A^{i-1, v-2} B & A^{i-1, v-3} B & \cdots & A^{i-1,0} B & 0 & \cdots & A^{0, v-2} B & A^{0, v-3} B & \cdots & B & 0 \\
A^{i-1, v-1} B & A^{i-1, v-2} B & \cdots & A^{i-1,1} B & A^{i-1,0} B & \cdots & A^{0, v-1} B & A^{0, v-2} B & \cdots & A^{0,1} B & B
\end{array}\right] .
$$

matrix. Furthermore, in the sequel it is shown that the matrix in (27) has more to do with the local controllability of the local states of the 2D system in (1). Note that, in the following of this section, it is assumed that $\mu \geq n$ and $v \geq n$.

Lemma 4: System (17) is controllable at kth (for $k=$ $1, \cdots, \mu)$ step with unconstrained control inputs $U$, if and only if the $\operatorname{rank}\left(\mathscr{C}_{k} \cdot \mathscr{C}_{k}^{T}\right)=v \cdot n$.

Proof: It is seen that the $k t h$ step controllability matrix of (17) is equal to $\mathscr{C}_{k}$ from Lemma 3 and, hence, this system is controllable if and only if $\mathscr{C}_{k}$ has full row rank.

Moreover, in the following theorem it will be shown that when $\mu \geq n, v \geq n$ and matrix $\mathscr{C}_{\mu}$ is of full row rank, the local controllability matrix $C_{n n}$, and hence, $C_{\mu v}$ will be of full row rank. However, the converse of this issue is not always true.

Theorem 1: The local controllability matrix $C_{n n}$ has full row rank if the matrix $\mathscr{C}_{\mu}$ has full row rank where $\mu \geq n$ and $v \geq n$.

Proof: As seen, the matrix $\mathscr{C}_{\mu}$ has $v$ row blocks with each block of dimension $\{n \times(\mu \cdot v \cdot m)\}$. It is not hard to show that the nonzero blocks of $n t h$ row block of the matrix $\mathscr{C}_{\mu}$ is equal to the controllability matrix $C_{\mu n}$. Hence, if $\mathscr{C}_{\mu}$ has full row rank, $C_{\mu n}$ has full row rank. From Lemma 1 2D system (1) is locally controllable in a given rectangle $[(0,0),(\mu, v)]$. According to Lemma 2 , it can be concluded that $C_{n n}$ is of full row rank.

In other words, whenever the matrix $\mathscr{C}_{\mu}$ has full row rank the $1 \mathrm{D}$ form system (17) is controllable and the 2D system (1) is locally controllable in a given rectangle $[(0,0),(\mu, v)]$ with unconstrained control inputs. Besides, the previous theorem can be confined to the following form. Now comes the main result of this section.

Theorem 2: The 1D form (17) of 2D system (1) is controllable if and only if the matrix pair $\left(A_{2}, B\right)$ is controllable.

Proof: Firstly, it is clear that the nonzero blocks of the first row block of matrix $\mathscr{C}_{n}$ is equal to the controllability matrix $C_{n 1}$. If $\mathscr{C}_{n}$ has full row rank, the matrix $C_{n 1}$ is of full row rank. Moreover, the nonzero blocks of $C_{n 1}$ is as

$$
\begin{aligned}
& {\left[A^{n-1,0} B|\cdots| A^{1,0} B \mid A^{0,0} B\right]} \\
& =\left[A_{2}^{n-1} B|\cdots| A_{2} B \mid B\right] .
\end{aligned}
$$

It is well known that the right hand side of the above equation is equal to the controllability matrix of the pair $\left(A_{2}, B\right)$. Conversely, suppose $\left(A_{2}, B\right)$ is controllable. It is known that matrices $\hat{K}$ and $\hat{L}$ are block lower triangular matrices as in (35) shown at the top of the next page. Hence, the pair $(\hat{K}, \hat{L})$ is controllable if there exists a state-feedback matrix
$F$ such that all the eigenvalues of the term $\hat{K}+\hat{L} F$ are freely assigned. Define the state-feedback $F$ as the following block diagonal matrix,

$$
F=\operatorname{diag}\left(F_{1}, \cdots, F_{v}\right)
$$

where $F \in R^{[v \cdot m] \times[v \cdot n]}$ and $F_{i} \in R^{m \times n}, 1 \leq i \leq v$. Now, it can be seen that $\hat{K}+\hat{L} F$ is as (37) at the top of the next page where $\{*\}$ means irrelevant entries. Thus, it is obvious that in the case that $\left(A_{2}, B\right)$ is controllable, all the eigenvalues of the diagonal blocks of $\hat{K}+\hat{L} F$ can be freely assigned by the state-feedbacks $F_{i}(1 \leq i \leq v)$. As a result, it can be seen that the controllability of $\left(A_{2}, B\right)$ suffices that $(\hat{K}, \hat{L})$ is controllable as well.

Here, according to Theorem 2, a new notion of controllability for $2 \mathrm{D}$ systems is defined.

Definition 2: 2D system in (1) is said to be directionally controllable with respect to the direction $\{j\}$, if its 1D form $\Sigma_{v}$ in (17) is controllable.

Proposition 1: 2D system in (1) is directionally controllable with respect to the direction $\{j\}$ if, and only if, the matrix pair $\left(A_{2}, B\right)$ is controllable.

\section{Directional controllability with respect to $\{i\}$-direction}

In the procedure of [9] and this paper, it is assumed that the $\{j\}$-direction is finite, and hence, the local states located in the same $\{j\}$-direction form the 1D stacking vectors. In the case that the $\{i\}$-direction is of finite dimension, the local states located in the same $\{i\}$-direction can be stacked to form the 1D stacking vectors. Similarly, a sufficient and necessary condition of the directional controllability with respect to $\{i\}$-direction can be obtained as follows.

Proposition 2: The 2D system in (1) is directionally controllable with respect to the direction $\{i\}$, if and only if the matrix pair $\left(A_{1}, B\right)$ is controllable.

\section{NumericAl ExAMPLE}

Consider the following 2D first FM model

$$
\begin{gathered}
A_{1}=\left[\begin{array}{cr}
-0.56 & 0 \\
0 & 0
\end{array}\right], A_{2}=\left[\begin{array}{ll}
0.33 & -0.54 \\
1.26 & -0.41
\end{array}\right], \\
A_{0}=\left[\begin{array}{rr}
0.51 & -0.09 \\
0.00 & 0.04
\end{array}\right], B=\left[\begin{array}{l}
0 \\
2
\end{array}\right] .
\end{gathered}
$$

Here $x \in R^{2}$ and $u \in R$. We assume this 2D system over the rectangle $\mu \times v(\mu=5$ and $v=3)$. Furthermore, it is supposed 


$$
\hat{K}=\left[\begin{array}{ccccc}
A_{2} & 0 & \cdots & 0 & 0 \\
A_{1} A_{2}+A_{0} & A_{2} & \cdots & 0 & 0 \\
\vdots & \vdots & & \vdots & \vdots \\
A_{1}^{v-1} A_{2}+A_{1}^{\nu-2} A_{0} & A_{1}^{v-2} A_{2}+A_{1}^{v-3} A_{0} & \cdots & A_{1} A_{2}+A_{0} & A_{2}
\end{array}\right], \hat{L}=\left[\begin{array}{ccccc}
B & 0 & \cdots & 0 & 0 \\
A_{1} B & B & \cdots & 0 & 0 \\
\vdots & \vdots & & \vdots & \vdots \\
A_{1}^{v-1} B & A_{1}^{v-2} B & \cdots & A_{1} B & B
\end{array}\right] .
$$

$$
\hat{K}+\hat{L} F=\left[\begin{array}{ccccc}
A_{2}+B F_{1} & 0 & \cdots & 0 & 0 \\
* & A_{2}+B F_{2} & \cdots & 0 & 0 \\
\vdots & \vdots & \ddots & \vdots & \vdots \\
* & * & \cdots & A_{2}+B F_{v-1} & 0 \\
* & * & \cdots & * & A_{2}+B F_{v}
\end{array}\right]
$$

that

$$
\begin{gathered}
x(0, j)=\left[\begin{array}{l}
1 \\
1
\end{array}\right], \quad 0 \leq j \leq 3, \\
x(i, 0)=\left[\begin{array}{l}
0.2 \\
0.2
\end{array}\right], \quad 0 \leq i \leq 5 .
\end{gathered}
$$

It can be seen that despite the uncontrollability of the pair $\left(A_{1}, B\right)$, and, since the pair $\left(A_{2}, B\right)$ is controllable, the pair $(\hat{K}, \hat{L})$ is controllable. Moreover, $\mathscr{C}_{2}$ and $C_{22}$ have full row rank $\left(\operatorname{rank}\left(\mathscr{C}_{2}\right)=6\right.$ and $\left.\operatorname{rank}\left(C_{22}\right)=2\right)$. As a result, this 2D system can be said to be directionally controllable with respect to the direction $\{j\}$.

\section{CONCLUSiOns}

Controllability analysis of the 1D form of the underlying 2D systems is the main subject of this paper. To this end, firstly, a brief review of the recently proposed method to convert the first FM model to a 1D vectorial form using a row (column) process is given. This method applies to the 2D systems which one of their distinct variables propagates over finite horizon and thus can be stacked. Consequently, the original 2D system is replaced by a 1D virtual system which can be controlled easily. In this work, a state bi-diagonal block Toeplitz matrix is produced and it should be inverted to achieve the standard state-space model. More importantly, the controllability of the obtained $1 \mathrm{D}$ vectorial form and its relation to the controllability of the original first FM model is studied in this paper. A new notion of controllability named directional controllability is defined for $2 \mathrm{D}$ systems.

\section{REFERENCES}

[1] D.D. Givone and R.P. Roesser, Multidimensional linear iterative circuits, IEEE Transanctions on computers, vol. C-21(10), pp. 1067$1073,1972$.

[2] D.D. Givone and R.P. Roesser, Minimization of multidimensional linear iterative circuits, IEEE Transanctions on computers, vol. C22(7), pp. 673-678, 1973.

[3] E. Fornasini and G. Marchesini, State space realization theory of 2D filters, IEEE Trans. Aut. Contr., vol. 21, pp. 484-492, 1976.

[4] E. Fornasini and G. Marchesini, Doubly indexed dynamical systems, Math. Syst. Theory, vol. 12, pp. 59-72, 1978.

[5] W.A. Porter, and J.L. Aravena, 1-D model for M-D processes, IEEE, Trans. Circuits. and Systems, vol. CAS-31, no. 8, pp. 742-745, 1984

[6] C. Edwards, S. K. Spurgeon, Sliding Mode Control: Theory and Applications, Taylor and Francis, London, 1998.
[7] V. I. Utkin, Sliding Modes in Control Optimization, Springer, Berlin, 1992.

[8] J. Klamka. Controllability of 2D systems. The Fourth International Workshop on Multidimensional Systems, NDS 2005, pp. 199-206, 2005.

[9] A. Argha, L. Li and S.W. Su, A new approach to applying discrete sliding mode control to $2 D$ systems, 52th IEEE Conferences on Decision and Control, Florence-Italy, December 10-13, 2013.

[10] K. Galkowski, E. Rogers, and D.H. Owens. Matrix rank based conditions for reachability/controllability of discrete linear repetitive processes, Linear Algebra and its Applications, vol. 275, pp. 201-224, 1998.

[11] E. Rogers, K. Galkowski, and D.H. Owens. Control systems theory and applications for linear repetitive processes. vol. 349. Berlin: Springer, 2007.

[12] E. Rogers, K. Galkowski, A. Gramacki, J. Gramacki, and D.H. Owens Stability and controllability of a class of 2-D linear systems with dynamic boundary conditions. IEEE Transactions on Circuits and Systems I: Fundamental Theory and Applications, vol. 49, no. 2, pp. 181-195, 2002

[13] T. Kaczorek, Two-Dimensional Linear Systems, Springer-Verlag, 1985.

[14] M. Sebek, M. Bisiacco, and E. Fornasini, Controllability and Reconstructibility Conditions for 2-D Systems,, vol. 33, no. 5, pp. 496-499, 1988.

[15] T. Kaczorek, Local Controllability, Reachability, and Reconstructibility of the General Singular Model of 2-D Systems, IEEE Transactions on circuits and systems, vol. 37, no. 10, pp. 1527-1530, 1992.

[16] E. Fornasini, and M.E. Valcher, Controllability and Reachability of 2D Positive Systems: A Graph Theoretic Approach, IEEE Transactions on circuits and systems-I, vol. 52, no. 3, pp. 576-585, 2005.

[17] M. Bisiacco, On the state reconstruction of 2-0 systems, Syst. Contr Lett., vol. 5, pp. 347-353, 1985.

[18] M. Sebek, M. Bisiacco, and E. Fornasini, Controllability and reconstructibility conditions for 2-D systems, IEEE Trans. Automat. Contr., vol. 33, pp. 496-499, 1988.

[19] M. Bisiacco, State and output feedback stabilkability of 2-D systems, IEEE Trans. Circuit. Syst., vol. CAS-32, pp. 1246-1254, 1985. 